\title{
A Novel Way To Manage Acute Airway Obstruction
}

Holt Walters ${ }^{1}$, Okechukwu Okonkwo 1, Christopher Lobo ${ }^{2}$

Manchester University NHS Foundation Trust ${ }^{1}$, Royal Bolton Hospital ${ }^{2}$

\section{Introduction}

Parapharyngeal masses are rare and can result in airway compromise. Management is often complicated by their difficult anatomical location, and can require emergency airway management.

THRIVE is a novel technique for oxygenating patients using high flow humidified oxygen and shown to prolong apnoea times. It been suggested as a method to facilitate tracheostomy insertion in patients with complex airways. We discuss the management of parapharyngeal space masses and the complex airway using THRIVE.

\section{Case Report}

A 53-year-old male developed a spontaneous increase in rightsided neck swelling whilst weight lifting causing significant acute onset airway compromise. The patient's complex airway was managed using THRIVE. The lipoma was resected and is the largest parapharyngeal lipoma reported in the literature to date.

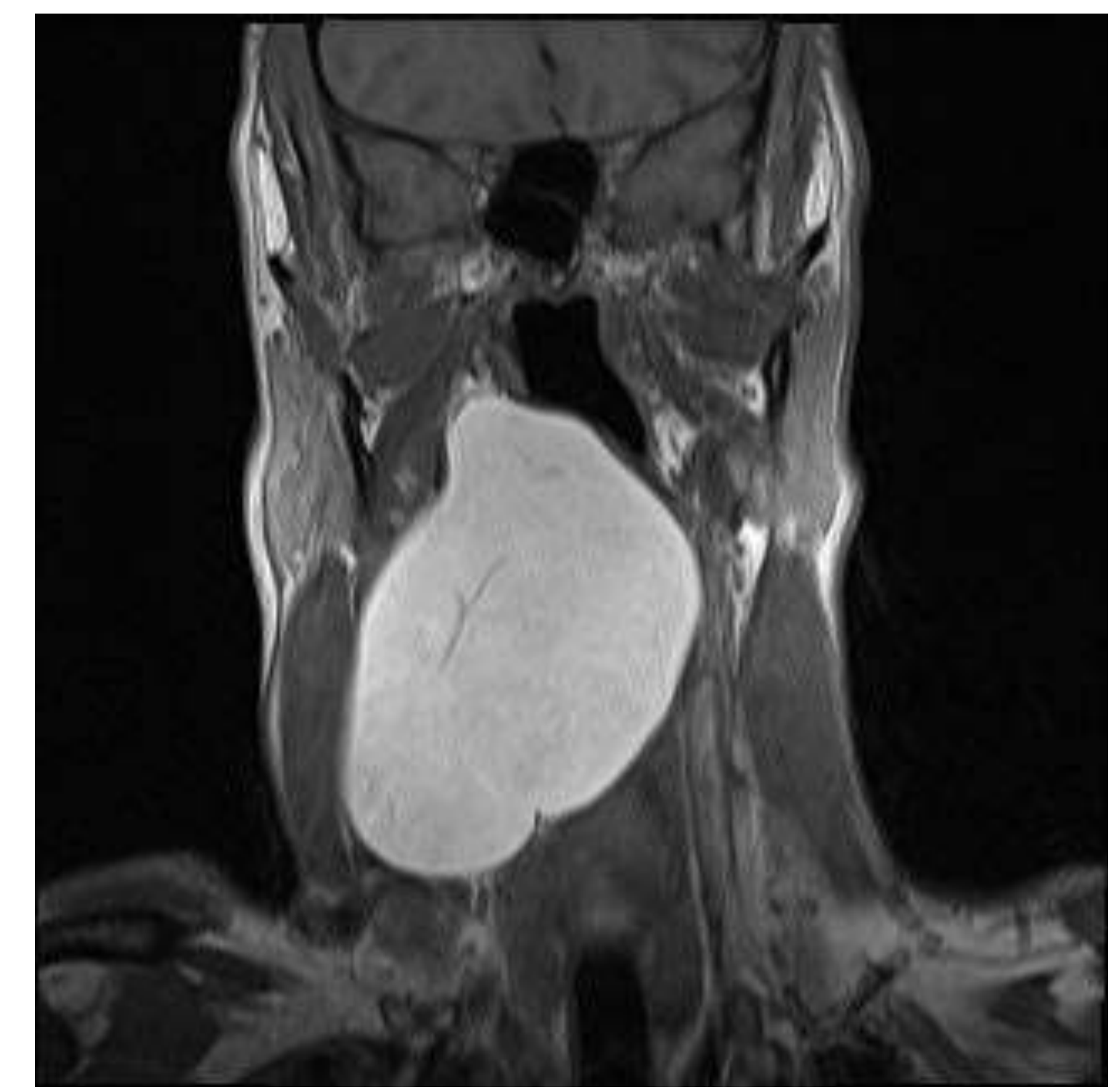

Fig. 1: MRI Head \& Neck showing a large parapharyngeal lipoma

\section{Discussion}

THRIVE is a novel technique for oxygenating patients using high flow humidified oxygen, based upon the phenomenon of aventilatory mass flow (AVMF). ${ }^{1}$

THRIVE has been shown to prolong apnoea times, and has been suggested as a method to facilitate tracheostomy insertion under sedation in patients who are unable to tolerate awake fibre-optic intubation.1,2

It extends the safe window of apnoeic oxygenation and increases the time available for interventions to secure the airway. The added benefit of allowing for patients to be sedated without the need for immediate intubation is the advantage of reducing the sympathomimetic effects stimulated by subsequent catecholamine secretion produced by discomfort and anxiety, carrying a secondary impact upon the level of bleeding experienced during surgery. ${ }^{2}$

These factors all allow a high pressure situation to become more controlled.

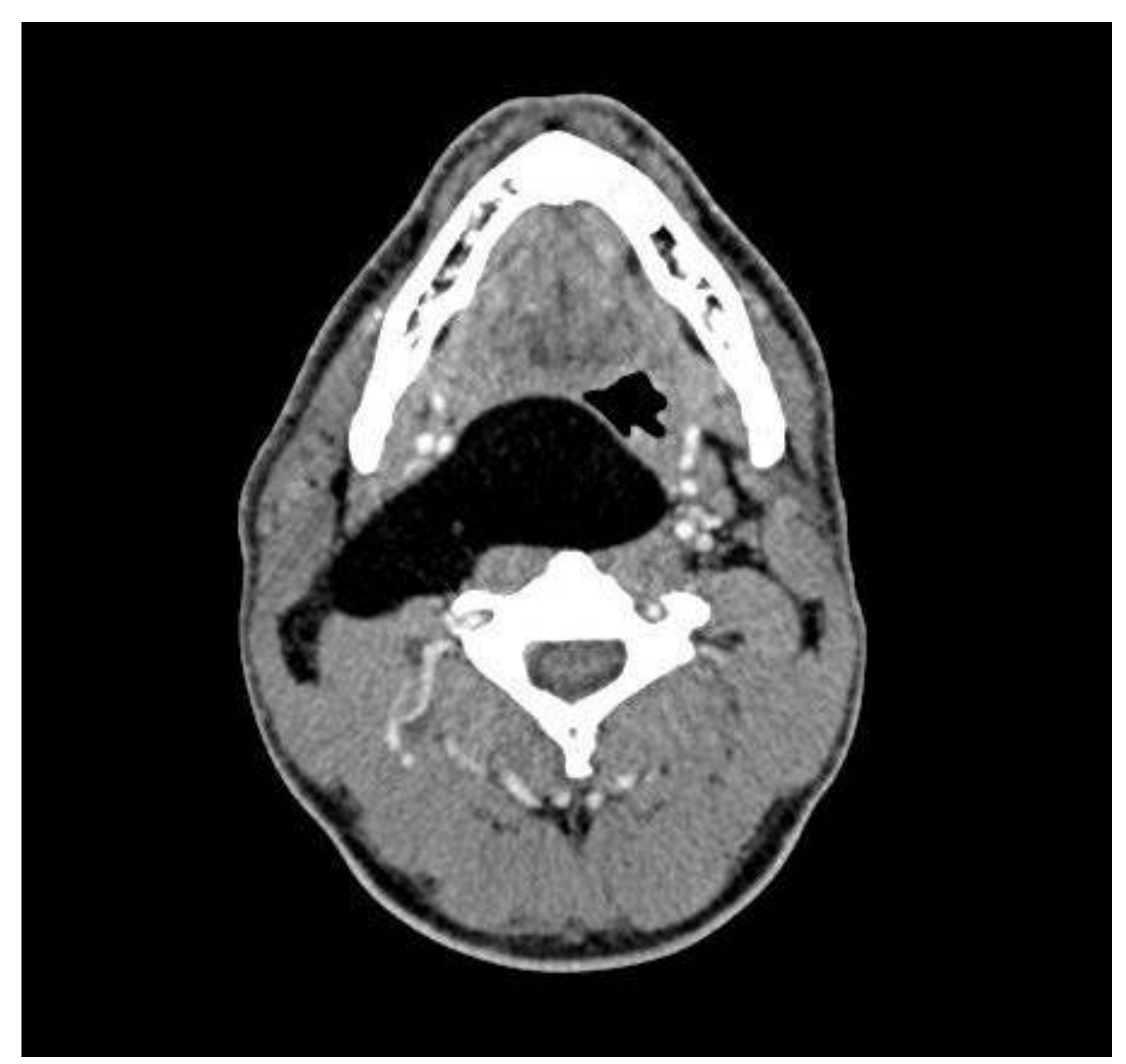

Fig. 2: CT Neck showing a large parapharyngeal lipoma

\section{Conclusion}

THRIVE has shown potential for facilitating emergency tracheostomy by extending apnoea times. This method of ventilating patients could revolutionise emergency airway management.

\section{References}

1. Patel A, Nouraei S. Transnasal Humidified Rapid-Insufflation Ventilatory Exchange (THRIVE): a physiological method of increasing apnoea time in patients with difficult airways. Anaesthesia 2015;70:323-329

2. Desai N, Fowler A. Use of Transnasal Humidified Rapid-Insufflation Ventilatory Exchange for Emergent Surgical Tracheostomy: A Case Report. A A Case Rep 2017;Nov1;9(9):268-270 